\title{
Melanocytic Lesions of the Nail Unit
}

\author{
Pembegül Güneş ${ }^{a} \quad$ Fatih Göktay ${ }^{b}$ \\ a Department of Pathology, University of Health Sciences, Haydarpaşa Numune Training \\ and Research Hospital, Istanbul, Turkey; ${ }^{\text {b}}$ Department of Dermatology, University of \\ Health Sciences, Haydarpaşa Numune Training and Research Hospital, Istanbul, Turkey
}

\author{
Keywords \\ Melanocytic neoplasms $\cdot$ Melanoma $\cdot$ Nails
}

\begin{abstract}
Melanocytic lesions of the nail unit are traditionally classified as follows: melanocytic activation, lentigo, nevi, and melanoma. Undoubtedly, melanoma is the most important malignant tumor of the nail unit. Differentiating nail unit melanoma from other melanocytic lesions is often difficult both clinically and histopathologically. This article will first describe the histology of the nail unit, followed by a brief summary of clinical and histopathological features as well as differential diagnosis of melanocytic lesions of the nail unit.
\end{abstract}

(c) 2018 The Author(s)

Published by S. Karger AG, Basel

\section{Histology}

For an ideal histopathological evaluation of melanocytic lesions, the normal histological structure of the different localizations of the nail unit should be known. The nail unit is composed of the nail matrix, nail bed, cuticle, nail folds and grooves, and fibrocollagenous supportive tissue [1, 2] (Fig. 1a). Some differences exist between the anatomical sites of the nail unit in terms of the number and functional activities of the melanocytes [1]. The number of melanocytes in the nail is low when compared to normal skin. In normal skin, the melanocyte count is $1,150 / \mathrm{mm}^{2}$, whereas the count in the nail matrix is $200-217 / \mathrm{mm}^{2}[1,3]$. In a 1-mm-long segment of the nail matrix, there are 4-9 (mean: 7.7) melanocytes [4]. Melanocytes located in the proximal nail matrix are inactive and do not produce melanin pigment. In the proximal matrix, the melanocyte count is $20 / \mathrm{mm}$, that is, $\sim 217 \pm 84 / \mathrm{mm}^{2}$. Approximately $50 \%$ of melanocytes located in the distal part of the nail matrix produce melanin. In this area, the melanocyte count is $132 \pm 34 / \mathrm{mm}^{2}$ and $4 / \mathrm{mm}$. The number of melanocytes in the nail bed is $45 \pm 25 / \mathrm{mm}^{2}$. Melanocytes of this area do not produce melanin pigment either $[3,4]$. 
Fig. 1. Normal nail matrix and bed. Longitudinal sections stained with H\&E. a The nail unit is composed of the nail matrix, nail bed, cuticle, nail folds and grooves, and fibrocollagenous supportive tissue. H\&E, $\times 4$. b The proximal matrix has 4-10 cell layers. $\mathrm{H} \& \mathrm{E}, \times 40$. c Immunohistochemistry with Melan-A demonstrates the melanocytes among basal epithelial cells; they are located suprabasally in the second to fourth cell layers from the base of the epithelium. H\&E, $\times 4$.
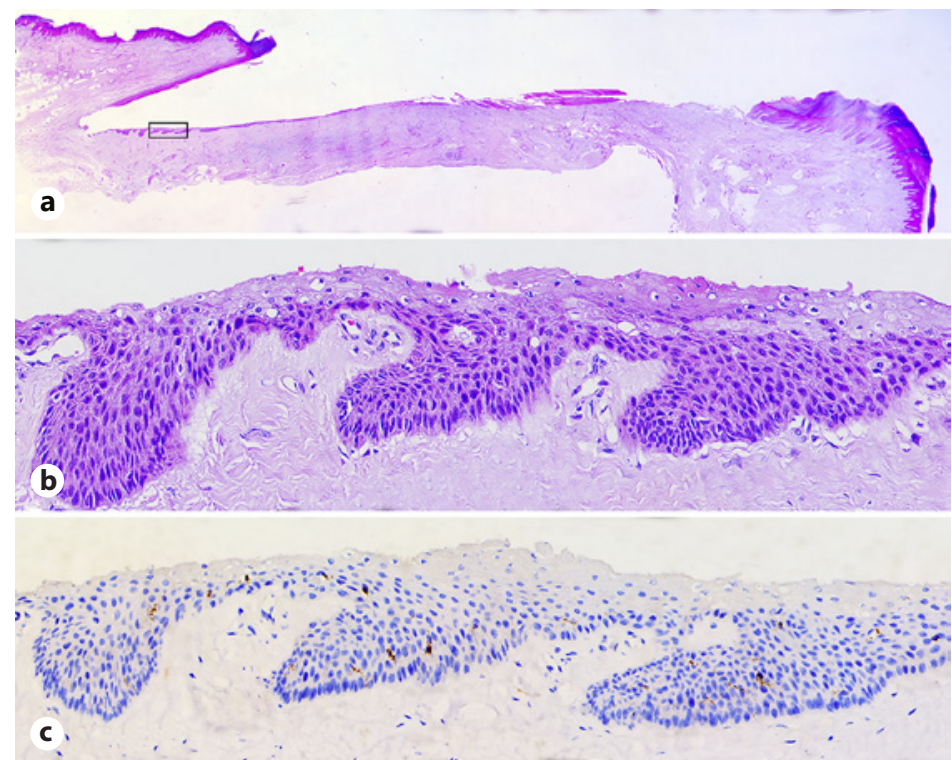

Melanocytes show some differences according to their anatomical localizations. The proximal matrix has the thickest epithelium in the nail unit. The basaloid epithelial thickness consists of 4-10 cell layers (Fig. 1b). Here, melanocytes can be seen in the upper part of the epithelium. They are located suprabasally in the second to fourth cell layers from the base of the epithelium (Fig. 1c). In the proximal matrix, melanocytes can only ascend to the prekeratogenous zone under the keratogenous zone. Conversely, the distal matrix epithelium consists of 2-3 cell layers, and melanocytes are localized in the first and second layers [1,4]. Melanocytes in the eponychium and nail bed are located in the basal layer. In the distal matrix, both the basaloid zone and the keratogenous zone are thin, and melanocytes are located in the basal layer [3]. Thus, it would be a pathological finding if the melanocytes appeared in the keratogenous zone. Since the proximal matrix melanocytes normally progress to the keratogenous zone, the presence of suprabasal dendritic melanocytes may cause diagnostic difficulty in cases of suspected melanoma. Melanocytes do not show pagetoid spread in the keratogenous zone. They appear as isolated cells without nest formation [3].

\section{Benign Melanocytic Lesions of the Nail Unit}

\section{Melanocytic Activation (Hypermelanosis)}

Melanocytic activation is the most frequent cause of benign melanonychia in adult patients. It may occur physiologically in dark-skinned persons. Melanonychia due to melanocytic activation may become evident in various conditions such as pregnancy, chronic trauma, nail biting, onychotillomania, occupational traumas, photochemotherapy, drug use, Addison disease, Nelson syndrome, Laugier-Hunziker syndrome, Peutz-Jeghers-Touraine syndrome, and vitamin B12 deficiency [1,4]. Macroscopically, the lesion appears as a longitudinal greyish pigmentation of the nail plate [5]. It can be observed as a periungual pigmentation in darkskinned patients. This finding should not be confused with Hutchinson's sign evident in melanoma lesions.

Melanocytic activation is characterized by hyperpigmentation of the matrix epithelium, secondary to activation of the suprabasal layer melanocytes in the proximal matrix and basal 

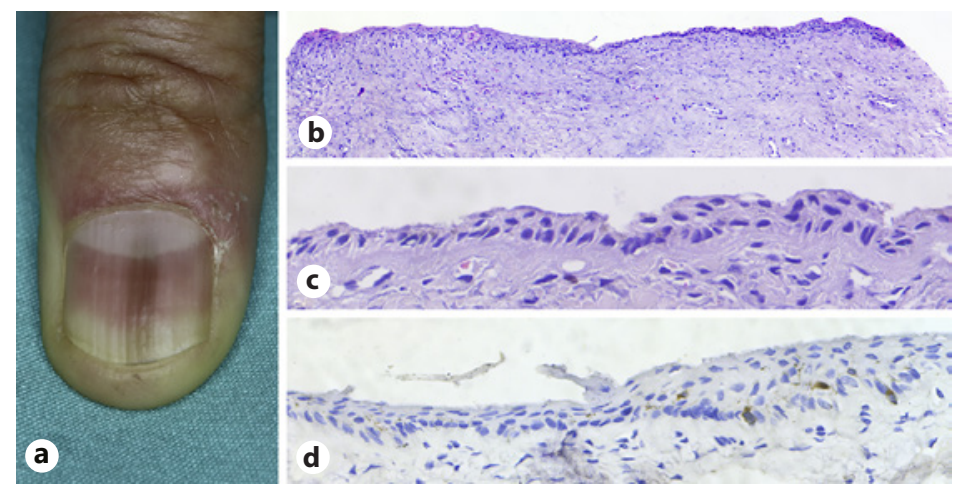

Fig. 2. Melanocytic activation. a A 49-year-old female patient with a 1.5-year history of longitudinal melanonychia located on her left hand thumbnail. Tangential matrix biopsy showed functional melanonychia. b H\&E stain showed some barely recognizable melanocytes and very little pigment and localized melanophages in the dermis. H\&E, $\times 4$. c Cytologically, the melanocytes were normal and no mitoses were seen. H\&E, $\times 40$. d Immunohistochemistry with Melan-A demonstrated the melanocytes among basal epithelial cells; they were located suprabasally in the first cell layers from the base of the epithelium without an increase in the number of melanocytes. Melan- $\mathrm{A}, \times 40$.

layer melanocytes in the distal matrix, without an increase in the number of melanocytes [1] (Fig. 2a). Cytologically, melanocytes have a normal appearance, and mitosis is not observed (Fig. 2b, c). Dendritic extensions of melanocytes are evident with accompanying dermal melanophages $[1,5]$.

In the biopsy specimens obtained from the nail matrix, epithelial hyperpigmentation cannot be demonstrated histopathologically by H\&E-stained sections. In many lesions, only a small amount of pigment can be displayed with Fontana-Masson staining. Histochemically, Fontana-Masson dye can be used to show pigment on both the nail plate and the matrix. Immunohistochemically, the normal number and structure of melanocytes can be shown by S-100, Melan-A, and HMB-45 antibodies (Fig. 2d). Histopathological examination does not provide a clue about the etiology of the disease [1].

The clinical and histopathological differential diagnoses of melanocytic activation include fungal infections, bacterial infections, and lentigo $[1,5]$. In the nail, the pigments other than melanin are not granular in appearance. Fungal pigmentation is yellowish-brown. It can be diagnosed histochemically by periodic acid-Schiff staining, which demonstrates the microorganisms in pseudohyphae and spore form. In bacterial infections, microorganisms can be detected by histochemical stains. As melanocytic activation and lentigo require different treatments, histopathological differentiation is important [1].

Histopathological evaluation should be performed by considering the normal number of melanocytes corresponding to the localization of the lesion [5]. If the number of melanocytes is close to normal and the melanocytes are located in the suprabasal layer, a diagnosis of melanocytic activation is favored.

\section{Melanocytic Hyperplasia (Lentigo)}

Melanocytic hyperplasia, also known as lentigo, is characterized by melanocyte proliferation. The lesion appears macroscopically as a brown pigmented longitudinal band [5]. The disease occurs predominantly in Caucasians and young adults.

Melanocytes are increased in number and abnormally located. Melanocytes double or triple the normal number without nest formation in the dermoepidermal junction. In a lentigo instance, 10-31 melanocytes per 1-mm segment of the lesion can be found [3] (Fig. 3a). 
Fig. 3. Melanocytic hyperplasia (lentigo). a A 29-year-old female patient with a 3-year history of longitudinal melanonychia located on her right hand second fingernail. b Tangential biopsy of a lentigo of the matrix showing heavily pigmented melanocytes in the matrix epithelium. $\mathrm{H} \& \mathrm{E}, \times 4$. c H\&E, $\times 40$. d Immunohistochemistry with HMB-45 demonstrated the melanocytes in the matrix epithelium. HMB- $45, \times 40$.
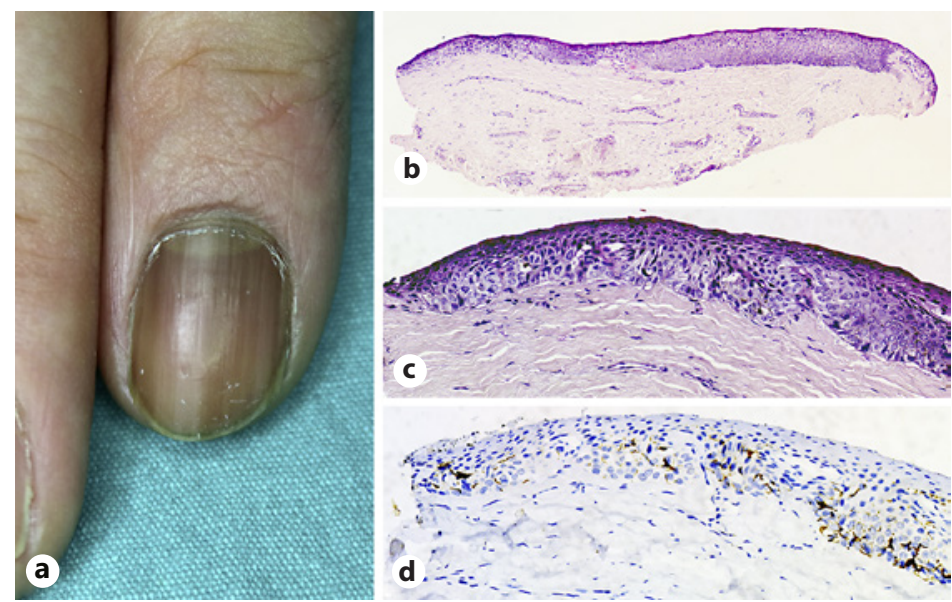

In light microscopy, full-thickness pigmentation is seen in both the matrix epithelium and the nail plate. Fontana-Masson staining is used to observe the fine granularity of the melanin pigment. There is no epithelial hyperplasia and pagetoid spread in the matrix [6]. A few melanophages may be found. Melanocytes align individually in the basal layer and may rarely be localized suprabasally (Fig. 3b, c). Melanocytes are usually normal, but they may show mild cytological atypia [2]. An increase in the number of melanocytes can be demonstrated by immunohistochemistry with S-100, Melan-A, and HMB-45 stains (Fig. 3d). S-100 positivity can be weak.

The differential diagnosis of this melanocytic proliferation includes melanocytic activation, melanocytic nevi, and melanoma. If the number of melanocytes is close to normal and melanocytes are localized suprabasally, a diagnosis of melanocytic activation is favored. In nevi, melanocytes proliferate in groups, forming nests, and not aligned as individual cells. There is no cytological atypia in melanocytes in lentigo [2]. Low cytological atypia may be seen in peripherally localized scattered and sparse melanocytes of melanoma. In such cases, lentigo cannot be distinguished from melanoma. Plump dendrites, diffuse pigmentation in the matrix epithelium, and pagetoid spreads of melanocytes should be interpreted favorably for malignancy [7].

\section{Melanocytic Nevi}

Melanocytic nevi are the most common cause of melanonychia in children [8, 9]. Clinically, a pigmented nail matrix nevus is observed as a longitudinal pigmented band on the nail plate. Although junctional nevi are the most frequent nevus subtype of the nail unit, blue nevi, Spitz nevi, and compound nevi can also occur in the nail unit on rare occasions $[1,4]$.

On microscopic examination of a melanocytic nevus, melanocytic proliferation is characterized by nest formation (Fig. 4a-d). The presence of different histological features in separate localizations of the nail unit may cause difficulty in evaluating the symmetry of the lesion. In melanocytic nevi of the nail unit, a lentiginous pattern, especially at the center of the lesion, may appear, along with a suprabasal pagetoid spread tendency, mild nuclear pleomorphism, nail plate involvement, and dermal inflammation nevi. The matrix nevus is usually a junctional and only rarely a compound nevus [1]. A periungual nevus is an infrequent condition. In this localization, the nevi can be junctional, compound, and dermal $[1,8]$. A blue nevus can originate from the nail matrix, nail bed, or periungual deep dermis, and it can be cellular or common. In the common type, a dark pigmented melanocytic proliferation occurs in the superficial dermis [10]. 
Fig. 4. Melanocytic nevi. a A 21-year-old female patient with a 2-year history of longitudinal melanonychia located on her left hand thumbnail. b Histopathology showed a junctional nevus of the matrix. H\&E, $\times 10$. c Melanocyte nests in the matrix epithelium. $\mathrm{H} \& \mathrm{E}, \times 40$. d Immunohistochemistry with Melan-A demonstrated the melanocytes nests in the matrix epithelium. Melan- $\mathrm{A}, \times 40$.
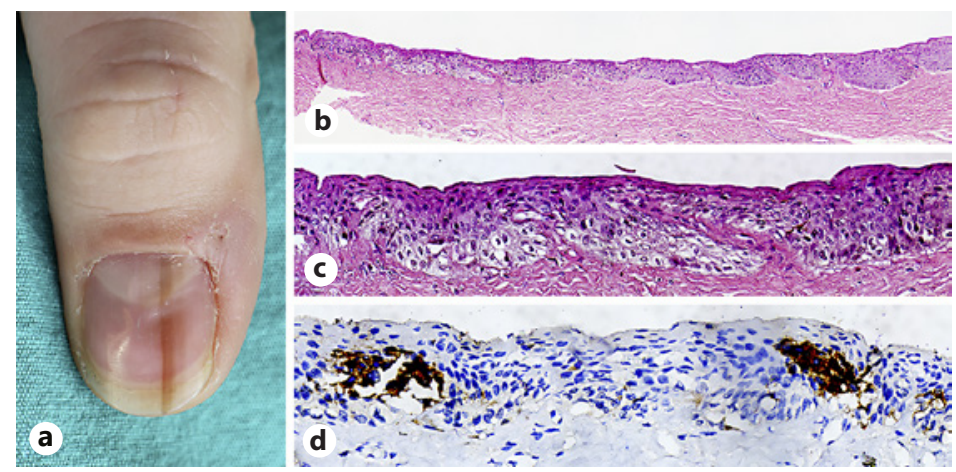

Determining the degree of atypia is somewhat subjective because the melanocyte nucleus is compared with the keratinocyte nucleus by locating its periphery. If the nuclear size of a melanocyte is equal to a keratinocyte nucleus, it is defined as minimal cytological atypia [11].

The presence of a pagetoid spread of individual atypical melanocytes should be regarded as favorable for atypical melanocytic proliferation [7]. An angular-shaped nucleus, dense chromatin, and polymorphism in different areas are important findings for melanoma [12]. Sometimes the cytological atypia presents in only a few cells, so clinical findings will be more important for the diagnosis and treatment approach [12].

The pyknotic nevus cells can be identified as melanin pigment [1]. The pigment reaches to the nail plate as it progresses in an oblique direction, which is the natural maturation direction of the matrix cells. Transcorneal elimination is seen in small melanocyte groups, especially in junctional nevi. Congenital nevi are deeply pigmented lesions showing deep dermal penetration.

The differential diagnosis of nail unit melanocytic nevi includes melanocytic activation, melanocytic proliferation, and melanoma [1]. The presence of nests is adequate to exclude the diagnosis of melanocytic activation [1]. A cellular blue nevus should be differentiated from melanoma metastasis [10]. Even if it is a focal finding, the presence of nuclear atypia and confluent proliferation in the dermoepidermal junction is diagnostic for melanoma. Although thick dendritic extensions of melanocytes and dermal inflammation support malignancy, inflammation may not be found in early-stage in situ melanomas. On the other hand, dendritic extensions can be seen in lentigo lesions. Coexistence of these findings is important for malignancy. Although subungual melanocytes numbering $>40 / \mathrm{mm}$ suggest the diagnosis of melanoma, this finding alone is not diagnostic [12].

\section{Subungual Melanoma}

Subungual melanomas (SUMs) constitute 1.5-2.5\% of all melanomas. SUMs usually occur in those aged 50-70 years and are very rare in children. Generally, SUMs develop de novo, and a benign melanocytic nevus does not precede their development [13]. Seventy percent of SUMs are seen in the thumb of the dominant hand and in the foot. The etiology of the disease is not known, but trauma can be an important factor [1]. The nail plate is a barrier that protects against UV penetration. However, this effect is weakened by factors such as absence of melanocytes, sebum, and stratum granulosum in the nail bed [14]. Since the matrix beneath the proximal nail fold protects against UV penetration, the pathogenesis of skin and nail melanomas has been considered different. Unlike skin melanomas, the role of UV penetration in a SUM is insignificant [15]. The cytokines produced by growth factors that are evoked during chronic inflammation play an important role in melanocyte proliferation and angiogenesis [16]. 
Therefore, SUMs are more common in black people and Asian people than skin melanomas [17]. Inspection, magnification, dermoscopy, and histopathology are the clinical approach steps to the diagnosis of SUM [17]. The presence of clinical findings such as longitudinal melanonychia $>5 \mathrm{~mm}$, first nail involvement, single nail involvement, enlargement of the pigmented band from distal to proximal, nail plate dystrophy, and periungual pigmentation are suggestive for malignancy [1]. Clinically, Hutchinson's sign, which is caused by spread of tumor cells to the proximal nail fold or the hyponychium, is important for the diagnosis of melanoma. However, it should be distinguished from pigmentation known as the pseudo-Hutchinson's sign due to melanocytic nevi, lentigines, squamous cell carcinoma in situ, trauma, radiation exposure, ethnic pigmentation, malnutrition, and hemorrhage [18].

Although longitudinal melanonychia is an important diagnostic clue because there is no melanin pigment in the nail bed, amelanotic melanoma constitutes up to $30 \%$ of nail apparatus melanomas [17]. Due to the lack of pigmentation, these cases can easily be confused with various nail disorders such as onychomycosis, warts, and pyogenic granulomas. This may cause a delay in the diagnosis of nail bed melanomas. As $66-75 \%$ of nail apparatus melanomas are pigmented, melanoma should be kept in mind if nail unit pigmentation is present [1].

Macroscopically abnormal pigmentation may be accompanied by nail plate atrophy and fissure. Since the tumor grows, nodule formation and ulceration can be seen. Atypical clinical forms are usually diagnosed later than these melanomas. In one study, the mean Breslow thickness was reported as $4.8 \mathrm{~mm}$ at diagnosis. Although most nail melanomas are pigmented, they can easily be overlooked by clinicians who are not experienced with nail disorders.

Although clinical experience is important, it is not enough for early diagnosis of melanoma. Some studies emphasize the necessity of performing biopsy and histopathological evaluation in all cases of clinically suspicious lesions for an accurate diagnosis of melanoma. In addition, although it is an invasive method, intraoperative dermoscopy has been deemed important both in diagnosing of the disease and in determining the course of the surgical procedure for suspected melanonychia cases [5].

SUMs are histologically classified into acral lentiginous melanoma, superficial spreading melanoma, and nodular melanoma subgroups [17]. However, most of the cases are of the acral lentiginous melanoma type [19]. The disease most commonly arises from the matrix and less frequently from the nail bed. Although many clinical criteria have been identified, histopathological examination is the diagnostic gold standard for SUM.

Histopathologically, the diagnostic criteria for in situ melanoma are infiltrative edge, increased intraepidermal melanocyte proliferation, increased suprabasal melanocytes, irregular distribution, and asymmetry of melanocytes, tendency of fusion in nests and epidermal consumption, cytological atypia, and dermal lymphoid cell infiltration [5] (Fig. 5a-d).

Detection of melanocytes in the nail plate corresponding to the matrix keratogenous zone is a very important finding for malignancy. A single intraungual melanocyte may be diagnostic for melanoma, but its absence does not exclude the diagnosis.

The diagnostic criteria for invasive melanoma of the nail are similar to those for the skin. Atypical melanocytic proliferation in the epidermis is accompanied by a dermal component that has lost its maturation. In addition, irregularly dispersed nests show increased cytological atypia and mitotic activity (Fig. 6a-d). Because of the lack of papillary dermis and subcutis in the nail apparatus, Clark's anatomical levels are different for skin melanomas. Therefore, the relationship between thickness and prognosis for nail apparatus melanoma is not similar to that in skin melanoma. Older age, ulceration, high mitotic index, amelanotic tumor, and advanced stage of disease are poor prognostic parameters [17]. The same AJCC staging system is used for both skin and nail melanomas. There are many important changes 

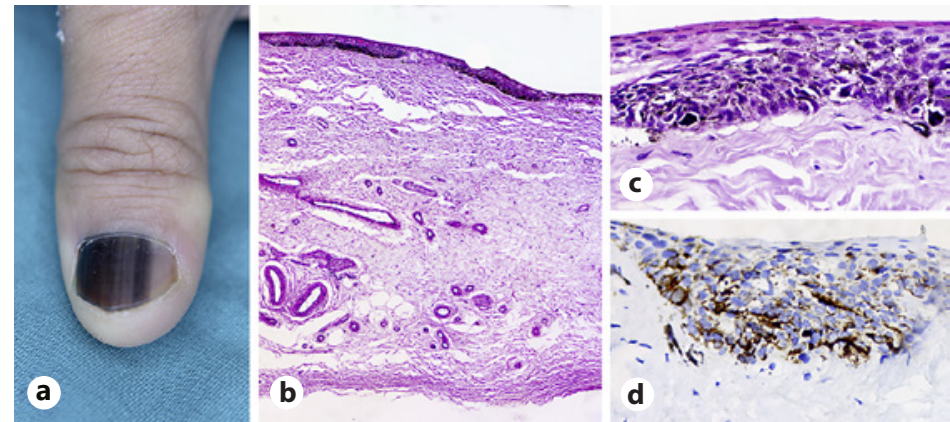

Fig. 5. Early in situ melanoma. a A 36-year-old male patient with a 6-year history of melanonychia located on his left hand thumbnail. See the black-brown pigmentation involving the whole nail plate area. $\mathbf{b}$ There was an increased density of solitary units of melanocytes displayed in a abnormal pattern at all layers of the subungual epithelium. Note the confluency of cells and pagetoid spread. Pigmentation was minimal. H\&E, original magnification $\times 4$. c There was an increased density of solitary units of atypical epithelioid melanocytes in the lower third of the subungual matrix epithelium. H\&E, original magnification $\times 40$. d Atypical melanocytes in the matrix epithelium were positive for HMB-45. Melan A, ×40.
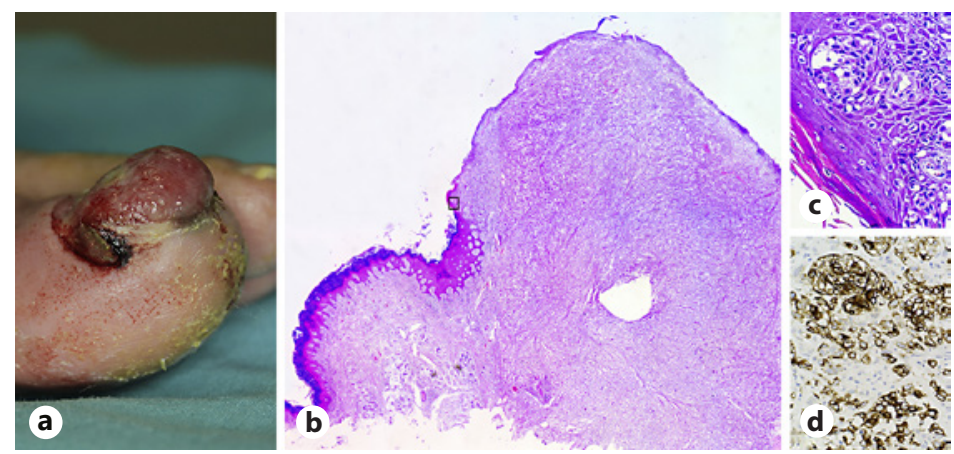

Fig. 6. SUM. a A 72-year-old male patient with a 6-month history of rapidly growing erythematous nodular lesion. $\mathbf{b}$ Invasive SUM showing the basal and suprabasal layers of the nail matrix and bed epithelium and dermis invaded by an excessive number of melanocytes often displaying severe cellular atypia. H\&E, $\times 4$. c In situ SUM displaying nests and lentiginous proliferation of severely atypical melanocytes. H\&E, $\times 40$. d Melanocytes are positive for HMB-45. HMB-45, ×40. SUM, subungual melanoma.

in the 8th edition of AJCC which can be summarized as follows: In the seventh edition, pT1 was subcategorized as a tumor with a thickness of $1 \mathrm{~mm}$, whereas it is revised in the eighth edition as $0.8 \mathrm{~mm}$. The mitotic rate is considered a prognostic factor in primary melanomas. However, it is not used as a staging criterion in the new edition. Previously, regional lymph node metastasis was described as "macroscopic" and "microscopic." In the eighth edition, it is restated as "clinically occult" and "clinically detected." In addition, in-transit, satellite, and microsatellite metastases are included in the $\mathrm{N}$ subcategory according to the number of tumor-involved nodes. The macroscopic extranodal involvement of a lymph node is no longer discarded as a criterion in the $\mathrm{N}$ staging. Finally, in the eighth edition, central nervous system metastasis is described as M1d, which did not exist in the seventh edition [20].

Immunohistochemistry is not useful in differentiating malignant lesions from benign ones. Melanocyte proliferation and migration can be evaluated with MART-1, Melan-A, Sox-10, MITF-1, and S-100 antibodies, which are widely used in practice. MART-1 and Melan-A are two different clones that are very close to each other. They show similar cytoplasmic staining. 
Pigment production continues in active melanocytes, and the presence of active dermal melanocytes detected by HMB-45 may be interpreted favorably for loss of maturation. HMB-45 and Melan-A are negative in 83\% of desmoplastic melanoma cases. In skin, S-100 may have a diagnostic value for desmoplastic melanoma, but S-100 staining may be lost in nail apparatus melanoma [1]. As Sox-10 and MITF-1 show nuclear staining, melanin pigment and melanosomes cannot be demonstrated by these stains. Therefore, their use is valuable in the presence of pigment. Sox-10 and MITF-1 nuclear positivity distinguish desmoplastic melanoma from fibroblastic and histiocytic mesenchymal tumors. These markers also differentiate melanocytes from keratinocytes. Since Ki-67 shows proliferative activity, it is valuable for differentiating between benign and malignant tumors [1].

Molecular analysis is necessary in metastatic and nonresectable tumors to determine their medical therapy. DNA mutations due to the oncogenic activation and inactivation of tumor suppressor genes play a role in melanoma development. Ckit mutation $(10-30 \%)$ is the most common genetic change with BRAFV600 (10-15\%) and NRAS (10-15\%) mutations in acral melanoma. BRAFV600 status is important in the selection of patients receiving BRAF and MEK inhibitors. Currently, NRAS inhibitors are used in phase 3 clinical trials and are not applied in routine practice. Ckit inhibitors are also available in the treatment of acral melanoma patients [21]. Molecular genetic changes are shown by fluorescence in situ hybridization and comparative genomic hybridization [22, 23].

Histopathological differential diagnoses include melanocytic proliferation, melanocytic nevi, and atypical melanocytic proliferation. It is important to know the localization of the biopsy for diagnosis. A diagnosis may be misleading in peripheral biopsies because the concentration of atypical melanocytes decreases in the periphery of the lesion in melanoma. The center of the lesion contains more diagnostic clues than the periphery. Additionally, cases in whom it is difficult to distinguish melanocytes and keratinocytes may also cause diagnostic difficulties. Florid, chaotic pagetoid spread of atypical cells, atypia, and hyperchromasia of melanocytes are more pronounced in melanoma than in melanocytic proliferation [18]. It should be noted that the focal pagetoid spread of melanocytic proliferation can be observed in benign entities. Although not common in nail melanomas, the presence of dermal inflammatory cells favors melanoma [1].

Melanin granules may be visible in melanocytic activation. Since the number of melanocytes is normal, the changes are slight. Additional immunohistochemical examination may contribute to the diagnosis. Lentigo lesions show increases in the number of melanocytes, melanin pigmentation, and melanophages in the dermis. One of the critical parameters in the differential diagnosis of melanocyte proliferation and in situ melanoma is a fourfold increase in melanocyte density in melanoma. The other criteria are the presence of multinucleation in melanocytes and inflammation in the in situ melanoma. Most of the matrix nevi are junctional and show a nest pattern. In melanoma, melanocytes form tight aggregates. The number of melanocytes is increased, and 39-136 melanocytes/mm are seen in the basal layer. Melanocytes have large and atypical nuclei and exhibit pagetoid spread. Presence of a single melanocyte in the nail plate is an important finding for malignancy. With in situ melanoma, irregular crowding and an increased number of melanocytes may be seen, but the cytological atypia is not prominent. Confluence and multinucleation in melanocytes (even if focal), basal lichenoid lymphoid infiltration, and migration of cell groups towards the surface are favorable for malignancy.

Nail discoloration in the hematoma cannot reach the free edge of the nail. In addition, as hemosiderin cannot be degraded by macrophages, it is histochemically negative for Prussian blue in subungual hematoma [1].

Up to $40 \%$ of nail diseases are caused by fungal microorganisms, which are often found in the differential diagnosis of melanoma. In the nail plate, the absence of staining with 

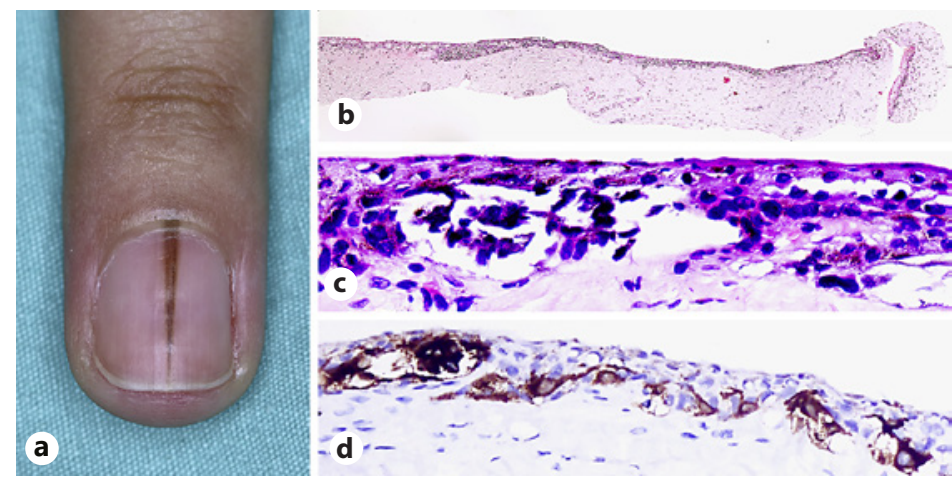

Fig. 7. Atypical melanocytic hyperplasia. a A 29-year-old female patient with a 2.5-month history of rapidly growing longitudinal melanonychia located on her left forth fingernail. $\mathbf{b}$ Tangential biopsy of atypical melanocytic hyperplasia exhibiting nests and lentiginous proliferation of melanocytes. H\&E, $\times 4$. c Atypical melanocytes in the matrix epithelium. H\&E, $\times 40$. d Melanocytes are positive for HMB-45. HMB-45, $\times 40$.

Fontana-Masson and the presence of periodic acid-Schiff-positive spores and hyphae are diagnostic for fungal infection [1].

Clinically, onychotillomania should be ruled out if there is loss of the thumbnail in a patient over the age of 40 . Subungual squamous cell carcinoma may destroy the nail plate. Making the distinction among amelanotic melanoma, subungual squamous cell carcinoma, and pyogenic granuloma is impossible without a biopsy. The presence of typical histological features is diagnostic for each lesion. In some cases, based on clinical appearance, the presence of warts, subungual fibrokeratoma, exocytosis, myxoid pseudocyst, and infections may be included in the differential diagnosis of the melanoma [1, 12, 17].

Several diagnostic challenges arise when differentiating atypical melanocytic proliferation from early in situ melanoma. Patterns of proliferation and pagetoid spread, cellular atypia, and accompanying interface inflammation may be slight and limited. In small biopsy findings, especially taken from the periphery of the melanoma where melanocyte density is low, melanocyte and keratinocyte differentiation cannot be made, and slight atypia is present. These features are known to be unfavorable for a definitive differentiation between these two entities. The term "atypical melanocytic hyperplasia" is used in these cases where a definitive distinction cannot be made [7, 24] (Fig. 7a-d).

Childhood melanocytic lesions are beyond the scope of this study due to the important differences from adult lesions in both clinical and histopathological aspects.

Generally, melanocytic nail lesions cause anxiety for both the patient and the physician. When dealing with such lesions, the essential tools and methods are patient history, detailed physical examination, biopsy from a suitable localization of the lesion when needed, and pathological examination considering the particular histological and pathological features of the nail apparatus. Additionally, rigorous clinical pathological correlation seems to be the most valuable approach for the diagnosis and treatment of melanocytic nail lesions. However, consulting with a nail expert will be inevitable in cases where diagnosis and treatment are difficult to determine.

\section{Acknowledgments}

The authors would like to thank Havva Karadağ Aluç for technical support and Ayşe Mine Önenerk for English language revision of the manuscript. 


\section{Statement of Ethics}

The authors have no ethical conflicts to disclose.

\section{Disclosure Statement}

The authors declare no potential conflicts of interest with respect to the authorship and publication of this article. They received no financial support for the research and authorship of this article.

\section{References}

1 Haneke E: Histopathology of the Nail: Onychopathology. Boca Raton, CRC Press, Taylor \& Francis Group, 2017.

2 Ruben BS: Pigmented lesions of the nail unit: clinical and histopathologic features. Semin Cutan Med Surg 2010;29:148-158.

3 Perrin C, Michiels JF, Pisani A, Ortonne JP: Anatomic distribution of melanocytes in normal nail unit: an immunohistochemical investigation. Am J Dermatopathol 1997;19:462-467.

4 Tosti A, Cameli N, Piraccini BM, Fanti PA, Ortonne JP: Characterization of nail matrix melanocytes with antiPEP1, anti-PEP8, TMH-1, and HMB-45 antibodies. J Am Acad Dermatol 1994;31:193-196.

5 Di Chiacchio N, Ruben BS, Loureiro WR: Longitudinal melanonychias. Clin Dermatol 2013;31:594-601.

6 Park JH, Lee DY, Kim N: Nail neoplasms. J Dermatol 2017;44:279-287.

7 Weedon D, Van Deurse M, Rosendahl C: "Occult" melanocytes in nail matrix melanoma. Am J Dermatopathol 2012;34:855.

8 Tosti A, Baran R, Piraccini BM, Cameli N, Fanti PA: Nail matrix nevi: a clinical and histopathologic study of twenty-two patients. J Am Acad Dermatol 1996;34:765-771.

9 Goettmann-Bonvallot S, Andre J, Belaich S: Longitudinal melanonychia in children: a clinical and histopathologic study of 40 cases. J Am Acad Dermatol 1999;41:17-22.

10 Gershtenson PC, Krunic A, Chen H, Konanahalli M, Worobec S: Subungual and periungual congenital blue naevus. Australas J Dermatol 2009;50:144-147.

11 McKee PH, Calonje E: Diagnostic Atlas of Melanocytic Pathology, ed 1. China, Elsevier, 2009.

12 Perrin C: Tumors of the nail unit. A review. Part I: acquired localized longitudinal melanonychia and erythronychia. Am J Dermatopathol 2013;35:621-636.

13 Phan A, Touzet S, Dalle S, Ronger-Savlé S, Balme B, Thomas L: Acral lentiginous melanoma: a clinicoprognostic study of 126 cases. Br J Dermatol 2006;155:561-569.

14 Baran R, Juhlin L: Drug-induced photo-onycholysis. Three subtypes identified in a study of 15 cases. J Am Acad Dermatol 1987; 17:1012-1016.

15 Mohrle M, Hafner HM: Is subungual melanoma related to trauma? Dermatology 2002;204:259-261.

16 Lesage C, Journet-Tollhupp J, Bernard P, Grange F: Post-traumatic acral melanoma: an underestimated reality? (in French). Ann Dermatol Venereol 2012;139:727-731.

17 Haneke E: Ungual melanoma - controversies in diagnosis and treatment. Dermatol Ther 2012;25:510-524.

18 Amin B, Nehal KS, Jungbluth AA, Zaidi B, Brady MS, Coit DC, Zhou Q, Busam KJ: Histologic distinction between subungual lentigo and melanoma. Am J Surg Pathol 2008;32:835-843.

19 Andre J, Sass U, Richert B, Theunis A: Nail pathology. Clin Dermatol 2013;31:526-539.

20 Amin MB, Greene FL, Edge SB, Compton CC, Gershenwald JE, Brookland RK, Meyer L, Gress DM, Byrd DR, Winchester DP: The Eighth Edition AJCC Cancer Staging Manual: Continuing to build a bridge from a population-based to a more "personalized" approach to cancer staging. CA Cancer J Clin 2017;67:93-99.

21 Carvajal RD, Antonescu CR, Wolchok JD, Chapman PB, Roman RA, Teitcher J, Panageas KS, Busam KJ, Chmielowski B, Lutzky J, Pavlick AC, Fusco A, Cane L, Takebe N, Vemula S, Bouvier N, Bastian BC, Schwartz GK: KIT as a therapeutic target in metastatic melanoma. JAMA 2011;305:2327-2334.

22 Curtin JA, Busam K, Pinkel D, Bastian BC: Somatic activation of KIT in distinct subtypes of melanoma. J Clin Oncol 2006;24:4340-4346.

23 Yeh I, Bastian BC: Genome-wide associations studies for melanoma and nevi. Pigment Cell Melanoma Res 2009;22:527-528.

24 Cho KH, Kim BS, Chang SH, Lee YS, Kim KJ: Pigmented nail with atypical melanocytic hyperplasia. Clin Exp Dermatol 1991;16:451-454. 